\title{
An Alternative Geometry for Quantum Cellular Automata
}

\author{
PAUL G. KRAUSE, RACHEL M. MUELLER, P. DOUGLAS TOUGAW* \\ and JANELLE M. WEIDNER
}

Department of Electrical and Computer Engineering, Valparaiso University, Valparaiso, IN 46383 USA

\begin{abstract}
We examine an alternative layout geometry for the quantum cellular automata (QCA) architecture. In the traditional QCA geometry, all of the cells are placed in a single plane, so that each cell interacts with a particular neighbor only along one of its edges. By rotating the cells out of the plane, we make it possible for neighbors to interact along all four edges at once. This increased interaction leads to a more bistable cell-cell response function and a $50 \%$ higher excitation energy. We also present a majority logic gate designed using three-dimensional cells.
\end{abstract}

Keywords: Quantum cellular automata, computer architecture, quantum-dot molecules

\section{INTRODUCTION}

The quantum cellular automata (QCA) architecture, which is composed of arrays of Coulombically coupled quantum dot molecules, is a revolutionary new paradigm for quantum computing [1]. QCA's offer new possibilities for computing at the nanometer scale, since they can be used to hierarchically implement any logical function [2-4].

In this paper, we present an alternative layout geometry for the QCA architecture by rotating the cells out of their plane. In the next section, we briefly review the QCA paradigm in two dimensions, while in Section 3 we examine the QCA architecture in three dimensions. By generalizing QCA cells to three dimensions and computing the resulting cell-cell response curve, we show that the three-dimensional cells are more bistable than the traditional two-dimensional cells.

In Section 4, we examine a three-dimensional majority logic gate. We show that two-dimensional and three-dimensional majority gates give identical functional results.

\section{TWO-DIMENSIONAL QCA DEVICES}

Figure 1 shows the basic building block in the QCA paradigm, the quantum dot molecule cell. Four quantum dots are spaced close enough for electrons to tunnel between neighboring pairs of dots. Two stable states are possible with this configuration. The first, corresponding to an

\footnotetext{
*Corresponding author: dtougaw@diamond.gem.valpo.edu.
} 


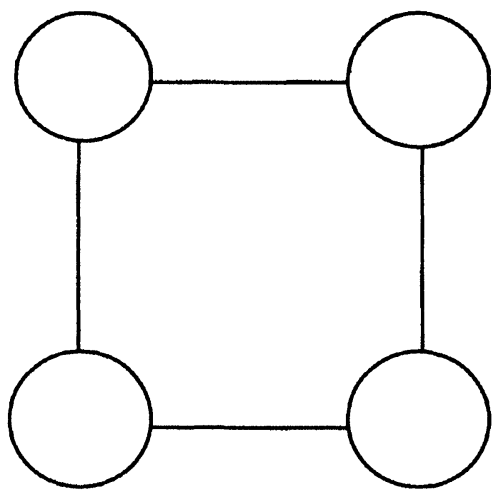

FIGURE 1 A schematic of the basic QCA cell. Electrons interact Coulombically and tunnel among the four sites.

electron occupying the upper right hand and lower left hand dots, represents $a$ polarization of +1 . The other, corresponding to an electron in the upper left hand and lower right hand dots, represents $a$ polarization of -1 . These two polarizations can represent binary values of " 1 " and " 0 ", respectively.

The Hamiltonian used to describe each cell includes Coulombic interaction between each pair of sites, near-neighbor tunneling, next-near-neighbor tunneling, on-site energies and on-site charging costs. The on-site energy of each site will be affected by the polarization of its neighboring cells.

Thus, Coulombic forces cause the polarization of one cell to affect the polarization of neighboring cells. The cell-cell response curve shown in Figure 2 is computed by solving the two-particle, timeindependent Schrödinger equation for the first cell in the presence of the second cell. The curve shows that the response of such cells is very bistable, which means that for even very small polarizations in one cell, the second cell is completely polarized. This feature is very attractive, since it allows these cells to encode a single binary bit in their quantum state. Furthermore, when propagating signals, the signal level is restored to the maximum polarization at every stage.

The most useful logic structure in the QCA paradigm is the three-input majority gate. A

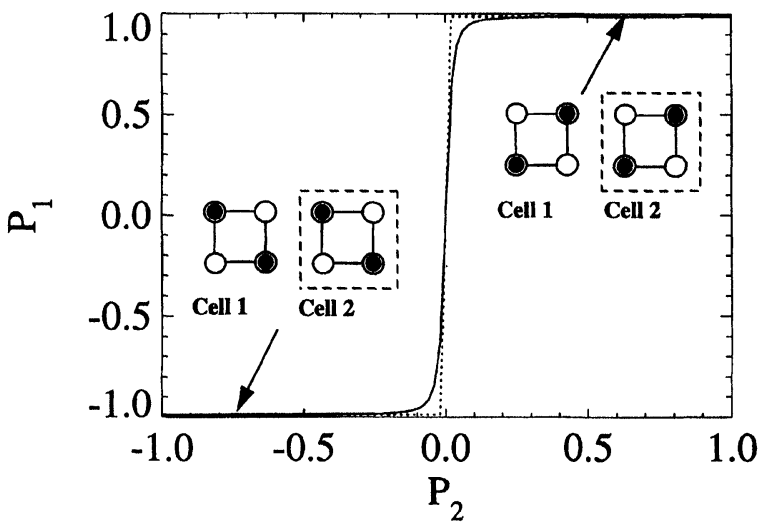

FIGURE 2 The cell-cell response function of two neighboring QCA cells. The bistable nature of the response function allows us to encode a binary bit in each cell.

majority gate is one in which the output agrees with the majority of the inputs. This logic structure is essential because we can produce both AND and OR gates from it. A two-input OR gate can be produced by setting one of the three inputs equal to 1 , while a two-input AND can be produced by setting one of the three inputs equal to 0 .

Figure 3 shows a schematic for the coplanar QCA majority gate. In this figure, inputs A, B and $\mathrm{C}$ are used as inputs. The output is propagated down the line of cells acting as a binary wire. The ground state of this multiple-cell structure was

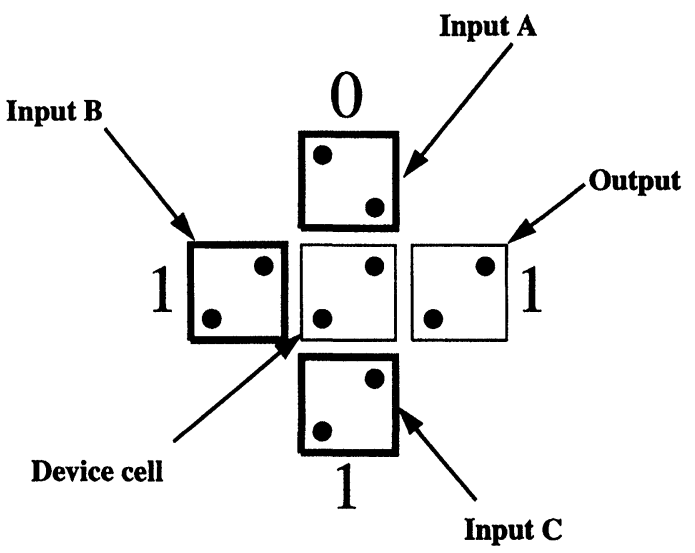

FIGURE 3 The coplanar majority voting gate. The device cell always matches a majority of the three fixed neighbors. 
computed by a self-consistent solution of the Schrödinger equation.

\section{AN ALTERNATIVE CELL GEOMETRY}

The QCA paradigm presented thus far has included only cells lying in a two-dimensional plane. By generalizing the architecture to three dimensions, we can show that the cell-cell interaction is significantly stronger than in the twodimensional case.

A schematic of our three-dimensional cell-cell layout is shown in Figure 4. Again, four dots are used, but in this case, the cells are facing each other so that all four of the dots are strongly interacting with the neighbor.

The cell-cell response curve of this layout is shown in Figure 5. The intercellular distance was computed such that the centers of the cells were at the same distance as the centers of the twodimensional calculation. The code to solve the time-independent Schrödinger equation was generalized to allow for Coulombic interactions in all three dimensions.

It should be noted that three-dimensional neighbors tend to align opposite one another, so the cell-cell response function was horizontally

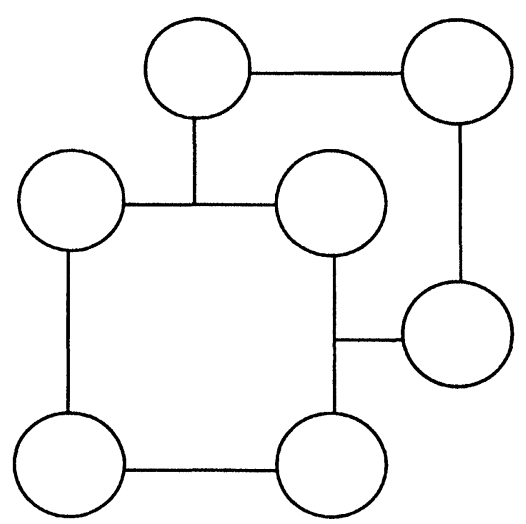

FIGURE 4 The cell layout for the three-dimensional cell-cell response function calculation.

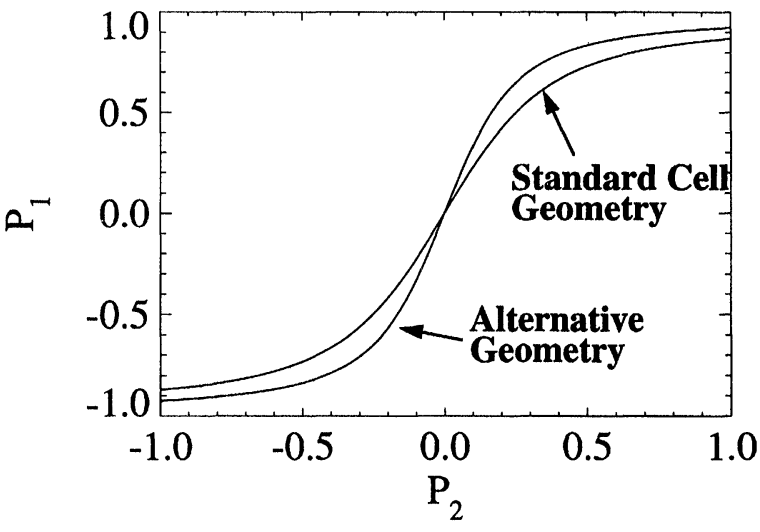

FIGURE 5 Cell-cell response function of the three-dimensional geometry. In addition to yielding a more bistable response function, use of the alternative geometry increases the excitation energy by $50 \%$.

flipped to allow easier comparison of the two curves. As with the "inverter chain" of the twodimensional QCA devices, this anti-alignment behavior can be overcome by using an even number of cells to transmit information. Of course, this will also make the implementation of three-dimensional inverters quite simple.

From the curves shown, it is easy to see that the three-dimensional curve is more bistable than the two-dimensional curve. In addition, the eigenvalue splitting between the ground state and the first excited state is $50 \%$ larger in the three-dimensional cells than it is in the two-dimensional cells. This is to be expected, since in the three-dimensional case, all four dots are Coulombically interacting, while in the two-dimensional case, only the nearneighbor dots strongly interact with each other.

Since the excitation energy between the ground state and the excited states is the quantity that controls the time-dependent evolution of the system, we can expect that this alternative geometry will propagate signals more quickly than the two-dimensional cells. In addition, the thermodynamic behavior, the adiabatic switching speed, and the maximum operating temperature are also dependent on the excitation energy, so similar improvements can be expected in those areas, as well. 


\section{A THREE-DIMENSIONAL MAJORITY GATE}

As mentioned in the previous section, the most important logical device of the QCA paradigm is the majority logic gate. Figure 6 demonstrates the layout of a QCA majority gate in three dimensions. Cells 1 and 3 are coplanar to cell 4 , so they tend to drive it to match their polarization. On the other hand, cell 2 drives cell 4 to an opposite polarity, since it interacts using the three-dimensional cell-cell response function. This can be cancelled by using an extra cell in that input, or it can be used to implicitly invert that input.

In order to ensure that the three inputs "vote" with equal weighting, the distances between cells were chosen such that when all four neighbors of the device cell are voting in opposite directions, the device cell exhibits no polarization.

To find this distance, the three coplanar cells (the device cell and two inputs) were placed a distance $60 \mathrm{~nm}$ apart from each other and the two coplanar drivers were set with $P=+1$. The other two neighbors were then set with $P=-1$ and moved away from the device cell until it exhibited zero polarization. At that point, we were certain that the horizontal and vertical neighbors were having the same impact on the polarization of the device cell. The distance between the non-coplanar cells at which this occurred was 1.7435 times larger

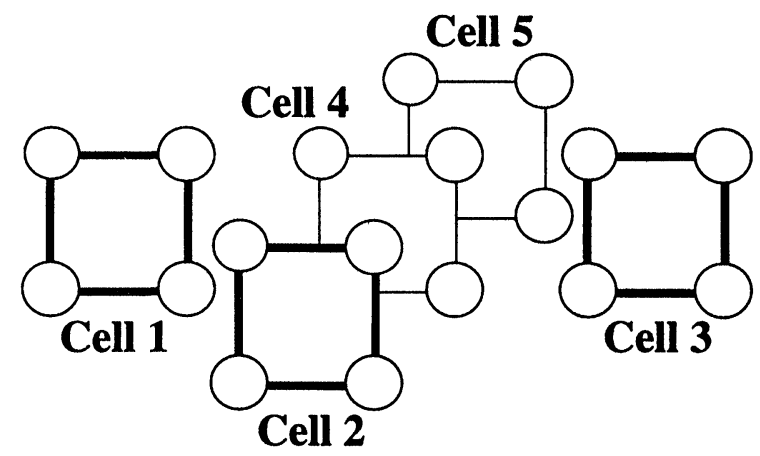

FIGURE 6 A schematic representation of the three-dimensional majority logic gate. Cells 1,2 and 3 are drivers, while cell 4 is the device cell and cell 5 is the output. Cells 2 and 4 tend to anti-align, so the input at cell 2 is implicitly inverted. than the distance between the coplanar cells, since the non-coplanar cells interact more strongly with each other.

We used the Intercellular Hartree Approximation to find the ground state of this multiple-cell system. The three input cells were fixed at a specific polarity (either +1 or -1 ), while the device cell and the output cell were allowed to change.

We tested each of the eight combinations of driver polarizations and found that in each case, the ground state corresponds to the correct result for a majority logic gate. The state of the output always matches the state of a majority of the three inputs, assuming the non-coplanar input has been implicitly inverted.

\section{CONCLUSIONS}

We have proposed an alternative geometry for the QCA computing architecture and we have shown that this geometry achieves a better cell-cell response than the two-dimensional implementation. We have also shown that an inverter and a majority logic gate can be constructed threedimensionally, so any combinational function can be constructed using this alternative geometry.

\section{Acknowledgements}

The authors would like to acknowledge the support of the Valparaiso University College of Engineering, as well as many productive conversations with the University of Notre Dame Nanoelectronic Devices Group.

\section{References}

[1] Lent, C. S., Porod, W. and Tougaw, P. D. (1993). Appl. Phys. Lett., 62, 714.

[2] Lent, C. S., Tougaw, P. D., Porod, W. and Bernstein, G. (1993). Nanotechnology, 4, 49.

[3] Tougaw, P. D., Lent, C. S. and Porod, W. (1993). J. Appl. Phys., 74, 3558.

[4] Lent, C. S. and Tougaw, P. D. (1993). J. Appl. Phys., 74, 6227. 


\section{Authors' Biographies}

Paul G. Krause is a graduate student at the University of Minnesota, where he is pursuing an advanced degree in Biomedical Engineering. He earned his B.S.E.E. degree from Valparaiso University.

Rachel M. Mueller is currently employed by Motorola, where she is working as a software engineer in the iDEN group. She earned her B.S.Cp.E. degree from Valparaiso University.
P. Douglas Tougaw is an assistant professor at Valparaiso University. He received his B.S.E.E. degree from Rose-Hulman Institute of Technology and his Ph.D. degree from the University of Notre Dame.

Janelle Weidner is currently an undergraduate student of electrical engineering at Valparaiso University. She is participating in a cooperative education experience with the National Security Agency. 

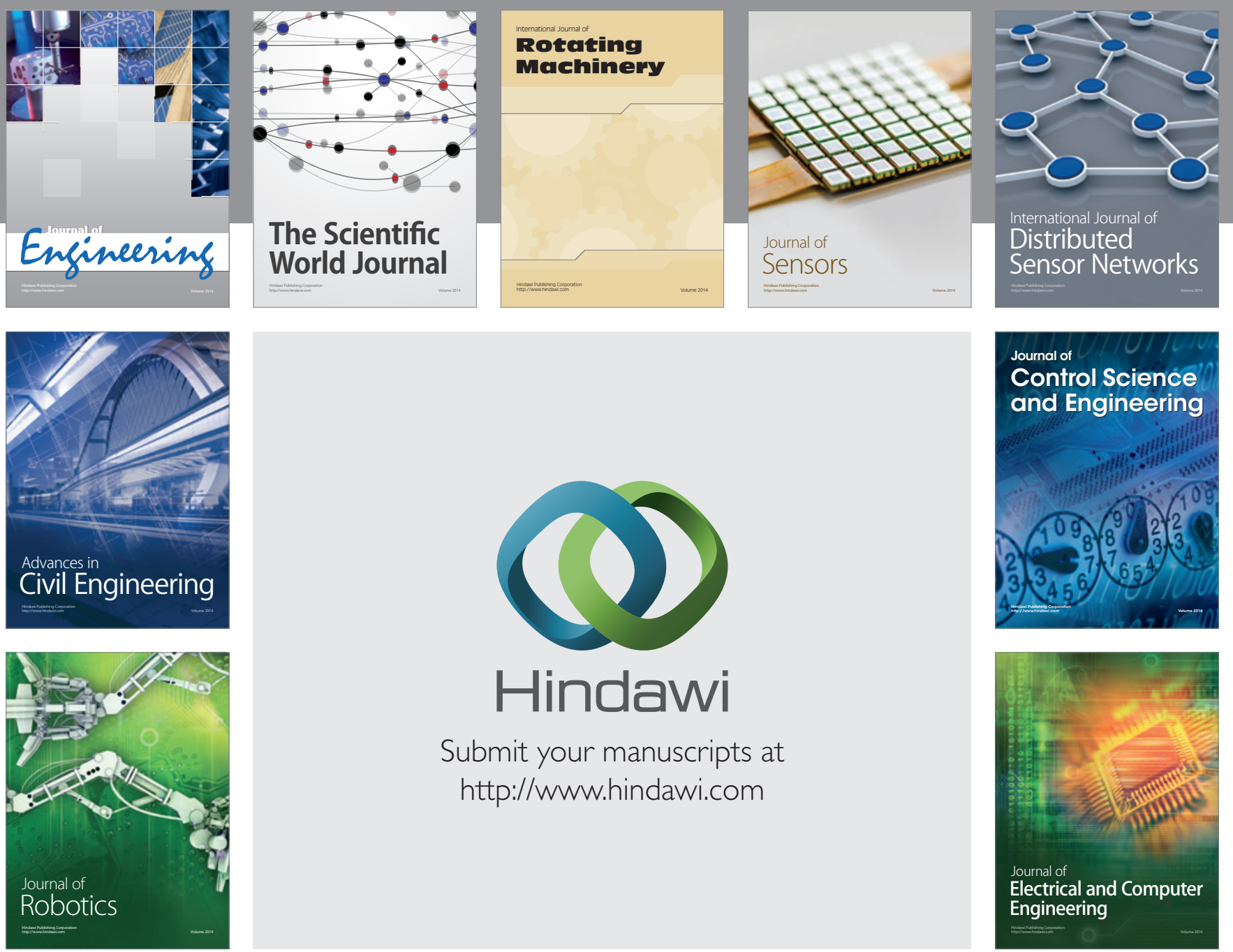

Submit your manuscripts at

http://www.hindawi.com
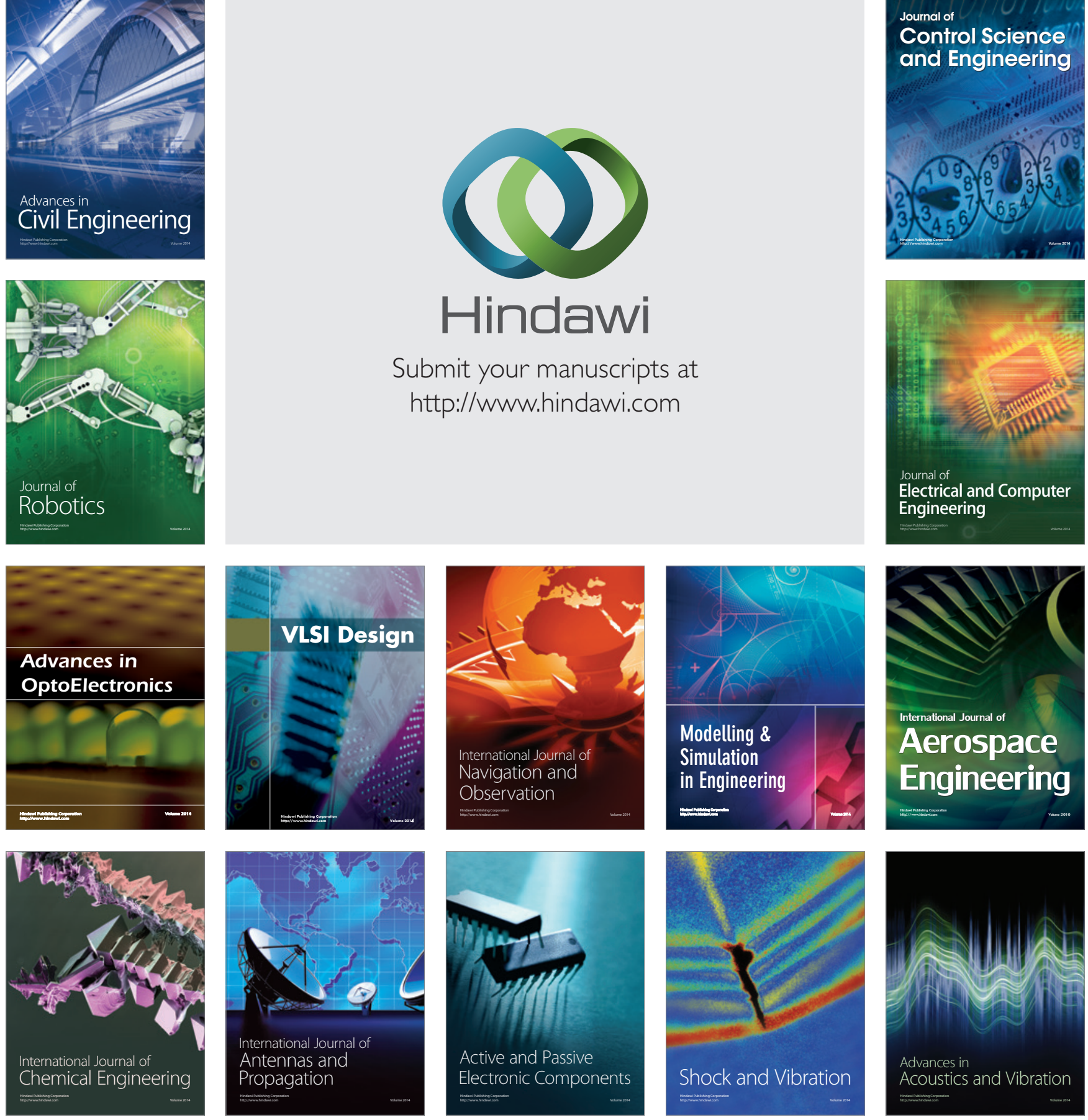